\title{
Biallelic Mutations in DNAJB11 are Associated with Prenatal Polycystic Kidney Disease in a Turkish Family
}

\author{
Esra Arslan Ateş ${ }^{\mathrm{a}} \quad$ Ayberk Turkyilmaz $^{\mathrm{b}}$ Kenan Delil ${ }^{\mathrm{c}}$ Ceren Alavanda ${ }^{\mathrm{c}}$ \\ Mehmet Ali Söylemez ${ }^{c}$ Bilgen Bilge Geçkinlic Pinar Ata ${ }^{c} \quad$ Ahmet Arman $^{c}$ \\ aDepartment of Medical Genetics, Marmara University Pendik Training and Research Hospital, İstanbul, Turkey; \\ ${ }^{b}$ Department of Medical Genetics, Karadeniz Technical University School of Medicine, Trabzon, Turkey; ${ }^{\circ}$ Department \\ of Medical Genetics, Marmara University School of Medicine, İstanbul, Turkey
}

\section{Established Facts}

- Polycystic kidney disease (PKD) is an inherited disease characterized by multiple cysts in the kidneys sometimes accompanied by liver cysts. The most common form of PKD is autosomal dominant PKD (ADPKD), having a prevalence of $0.4 \%$ in live births.

- A rarer form, autosomal recessive PKD (ARPKD), is seen in countries where consanguineous marriages are more frequent.

- The DNAJB11 gene, recently related to ADPKD, is located in chromosome 3q27 and encodes a soluble glycoprotein of endoplasmic reticulum.

\section{Novel Insights}

- DNAJB11 biallelic mutations may cause an antenatal severe form of ARPKD.

- The possibility of ARPKD due to biallelic mutations in ADPKD genes should be considered in genetic counseling.

- Bilaterally increased Virchow-Robin spaces, which indicate small vessel disease, in patients carrying monoallelic mutations in the DNAJB11 gene are a novel feature for this phenotype.

\section{Keywords}

DNAJB11 · Whole-exome sequencing · Nephrology · Genetic counseling $\cdot$ Polycystic kidney disease

\section{Abstract \\ Polycystic kidney disease (PKD) is a life-threatening condi- tion resulting in end-stage renal disease. Two major forms of PKD are defined according to the inheritance pattern. Auto-}

somal dominant PKD (ADPKD) is characterized by renal cysts, where nearly half of the patients suffers from renal failure in the 7th decade of life. Autosomal recessive PKD (ARPKD) is a rarer and more severe form presenting in childhood. Wholeexome sequencing (WES) analyses was performed to investigate molecular causes of the disease in the fetus. In this study, we present 2 fetuses prenatally diagnosed with PKD in a consanguineous family. WES analysis of the second fetus revealed a homozygous variant (c.740+1G>A) in DNAJB11
Correspondence to:

Esra Arslan Ateș, esraarslan.md@gmail.com 


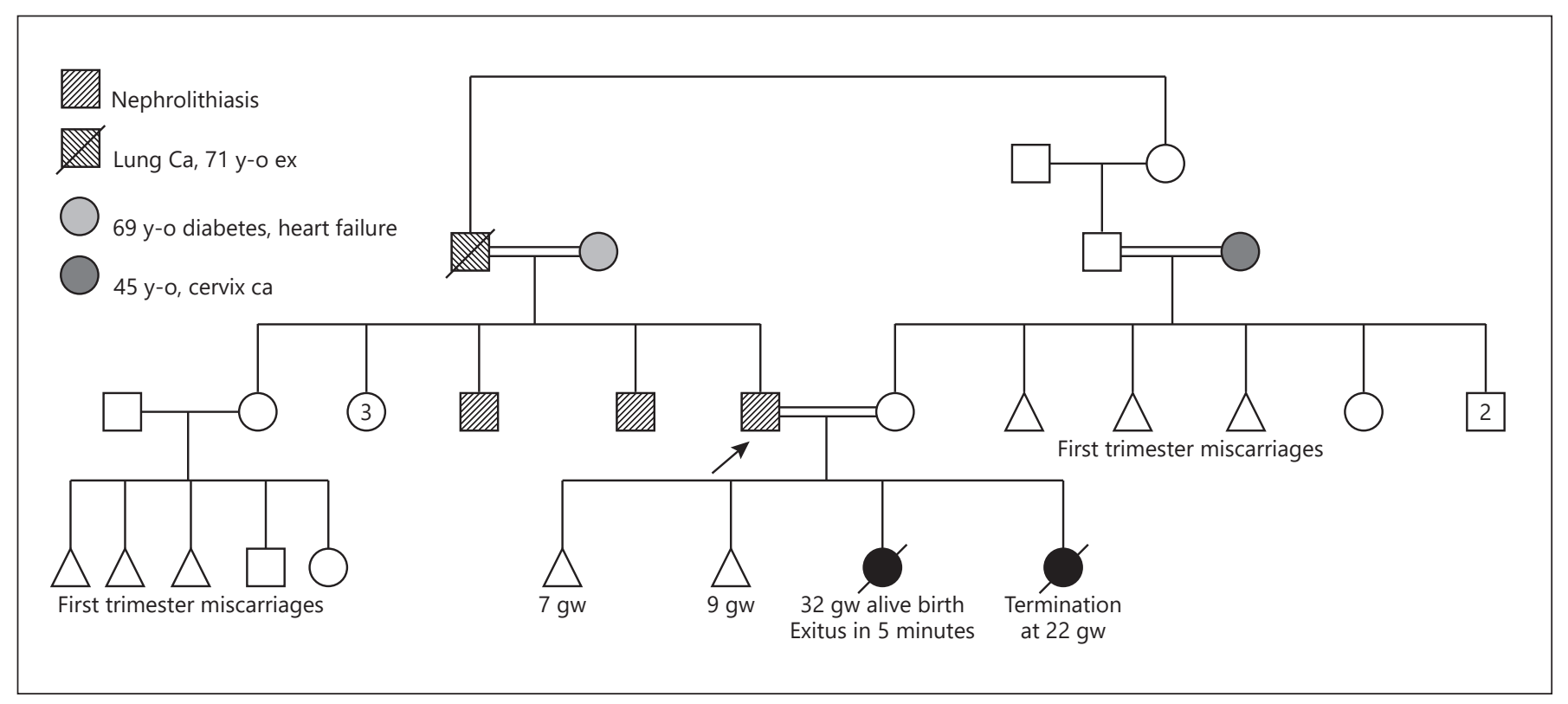

Fig. 1. Pedigree of the family.

which is related to ADPKD. This study reveals that DNAJB11 biallelic mutations may cause an antenatal severe form of ARPKD and contributes to understanding the DNAJB11-related ADPKD phenotype. The possibility of ARPKD due to biallelic mutations in ADPKD genes should be considered in genetic counseling.

(c) 2021 S. Karger AG, Basel

\section{Introduction}

Polycystic kidney disease (PKD) is an inherited kidney disease characterized by multiple kidney cysts and sometimes accompanied by liver cysts. The most common form of PKD is autosomal dominant PKD (ADPKD), with a prevalence of $0.4 \%$ in live births [Suwabe et al., 2020]. In OMIM, 4 genes (PKD1, PKD2, GANAB, DNAJB11) are listed as a cause of ADPKD [Mochizuki et al., 1996; Cornec-Le Gall et al., 2013, 2018; Porath et al., 2016]. A rarer form, autosomal recessive PKD (ARPKD) is seen in countries where consanguineous marriages are more frequent [Alzarka et al., 2017]. Biallelic PKHD1 and DZIP1L pathogenic variations are responsible for ARPKD [Bergmann et al., 2018]. Besides primarily related genes, also variations in genes associated with polycystic liver disease and ciliopathies are reported in PKD patients.

ARPKD presents more severely and at an earlier age of onset compared to ADPKD. ARPKD typically manifests perinatally or in childhood and the children die soon after diagnosis [Bergmann et al., 2018]. End-stage renal disease is expected in both forms, but in ADPKD renal functions remain normal for decades.

The DNAJB11 (DnaJheat shock protein family (Hsp40) member B11) gene, recently related to ADPKD, is located in chromosome 3q27 and encodes a soluble glycoprotein of endoplasmic reticulum (ER). It functions as a cofactor of binding immunoglobulin protein $(\mathrm{BiP})$ which is a chaperon located in ER and controls trafficking and folding of secreted and membrane proteins [Cornec-Le Gall et al., 2018]. Cornec-Le Gall et al. [2018] reported 7 ADPKD families carrying monoallelic DNAJB11 gene pathogenic variants and revealed the affect of dysfunctional protein product.

In this study, we report a prenatally diagnosed PKD case who was stillborn at the 22nd gestational week (gw) to healty consanguineous parents. Whole-exom sequencing (WES) analyses revealed a homozygous DNAJB11 variant segregating with the disease.

\section{Case Report}

A consanguineous couple was referred to our genetic diagnosis center due to bad obstetric history. The mother was a 25 -year-old with gravida 4 , who had 2 first-trimester miscarriages at the 7th and 9th gw, an a live-born at the 32nd gw, who died within 5 min after delivery (Case 1) and lastly an intrauterine death at the 22nd gw 
(Case 2). Autopsy of the Case 1 revealed polycystic kidneys, cardiomegaly, and pericardial effusion. There were no documented data about the first 2 miscarriages. In the last 2 pregnancies, bilateral infantile polycystic kidneys, cardiomegaly and pericardial effusion were detected at 20 th gw in level 2 ultrasonography examination. The last fetus also had overriding aorta, ventricular septal defect, and pulmonary artery was not visualized. In postmortem fetal dysmorphic examination a broad nasal bridge, bilateral epicanthus, bilateral simian creases, clinodactyly, bilateral pes equinovarus deformity, and normal external female genitalia were detected. There were no other skeletal anomalies nor polydactyly. Ultrasonography findings also confirmed internal organ malformations and no other abnormality was reported. Serologic and thirdtrimester biochemical screenings were normal. All were planned pregnancies with scheduled follow-up. Pedigree analysis revealed fourth-degree consanguinity between parents and father, and his 2 brothers had a history of recurrent nephrolithiasis. There were consanguineous marriages in the family with recurrent miscarriages which were not evaluated (Fig. 1). In the second case, prenatal conventional karyotyping and array-CGH analysis were normal.

\section{Materials and Methods}

For WES analysis, fetal DNA was extracted from umbilical cord blood with QiaAmpDNA mini kit (Qiagen Germantown, $\mathrm{MD}$, USA), all coding gene regions were amplified using Agilent SureSelect Human All Exon V5 kit (Agilent, Santa Clara, CA, USA). Prepared library was sequenced on the Illumina NovaSeq platform (Illumina Inc., San Diego, CA, USA), according to manufacturer guidelines. Data were analyzed using Sophia DDM ${ }^{\circledR}$ platform (Sophia Genetics SA, Switzerland). To call variants, sequencing data were aligned to human reference genome, hg19. Variants were filtered according to variant frequency among population studies (lower than 0.01 in 1000 Genomes, Exome Aggregation Consortium database, Exome Sequencing Project, Genome Aggregation Database), zygosity (variant fraction $>70 \%$ ), and coding consequence (synonymous, missense, nonsense, frameshift/in-frame, indel, splicing donor or acceptor site, intronic variants within 20 nucleotides from the exon/intron boundaries) (Fig. 2). The Human Gene Mutation Database (HGMD), ClinVar, and our in-house database were screened for detected variants. For prediction of pathogenicity of novel variants, in silico analysis tools including Provean, SIFT, MutationTaster, PolyPhen, and Human Splicing Finder (HSF 3.0) were used. For segregation analysis of candidate disease-causing variants, peripheral blood was obtained from parents and after DNA extraction targeted sequences were amplified using proper primers via PCR. The PCR products were sequenced via Illumina MiSeq platform (Illumina Inc.).

DNAJB11 Mutation in Prenatal Polycystic Kidney Disease

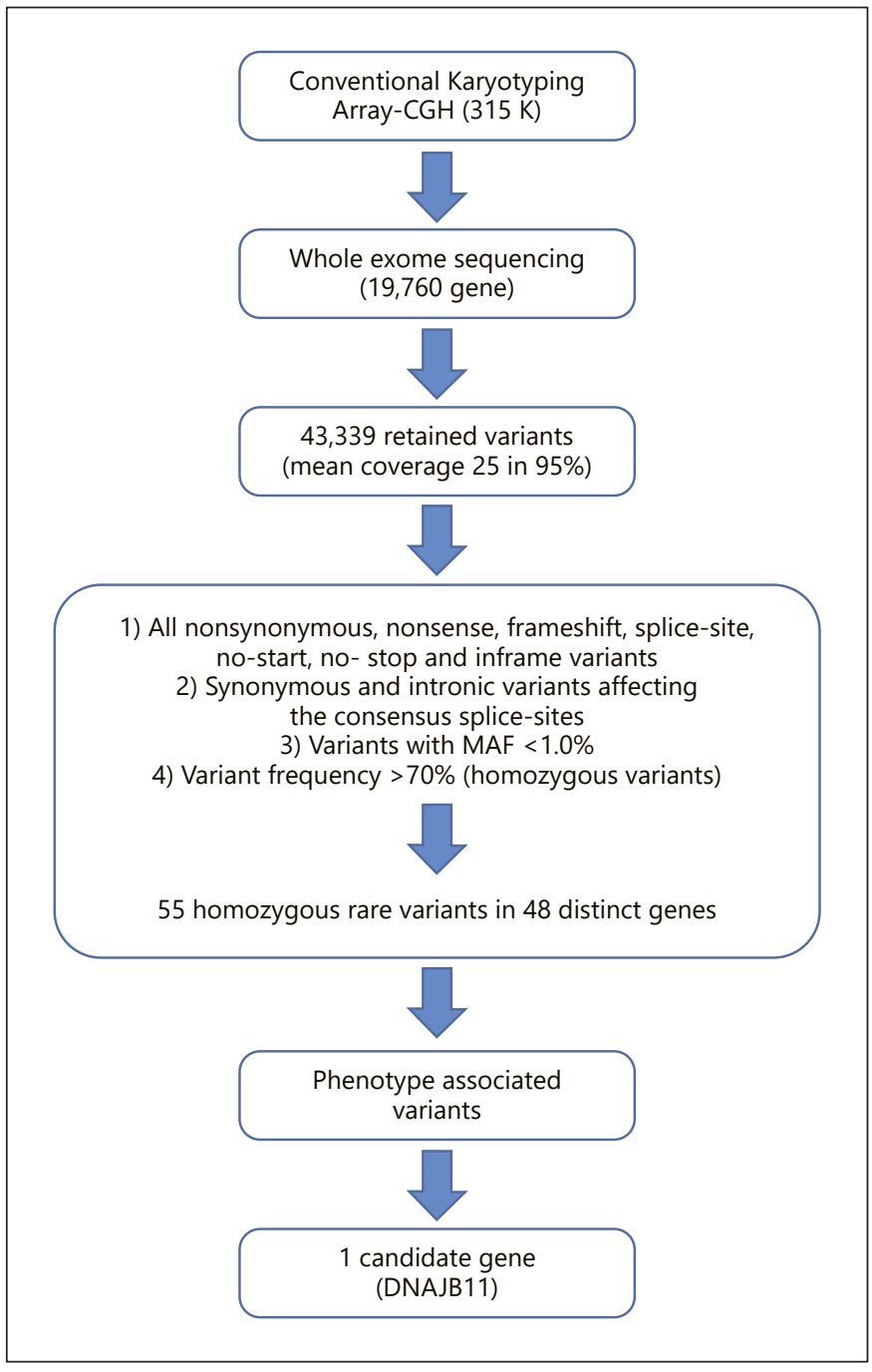

Fig. 2. Genetic testing algorithm.

\section{Results}

Clinical evaluation of the fetuses revealed PKD, which is lethal in the prenatal period and seems to be inherited in an autosomal recessive manner (Table 1). WES was performed from the cord blood of second fetus. Among the 43,339 variants detected, 55 homozygous rare variants in 48 distinct genes were retained after filtering the variants as described above. DNAJB11 was the only gene which was associated with a late-onset ADPKD previously [Cornec-Le Gall et al., 2018]. The other 47 genes were not involved in a PKD-associated pathway nor related to any kidney phenotypes.

The fetus carried a novel homozygous c.740+1G $>$ A variant in the DNAJB11 (RefSeq ID: NM_016306) gene 
Table 1. Clinical presentation of cases

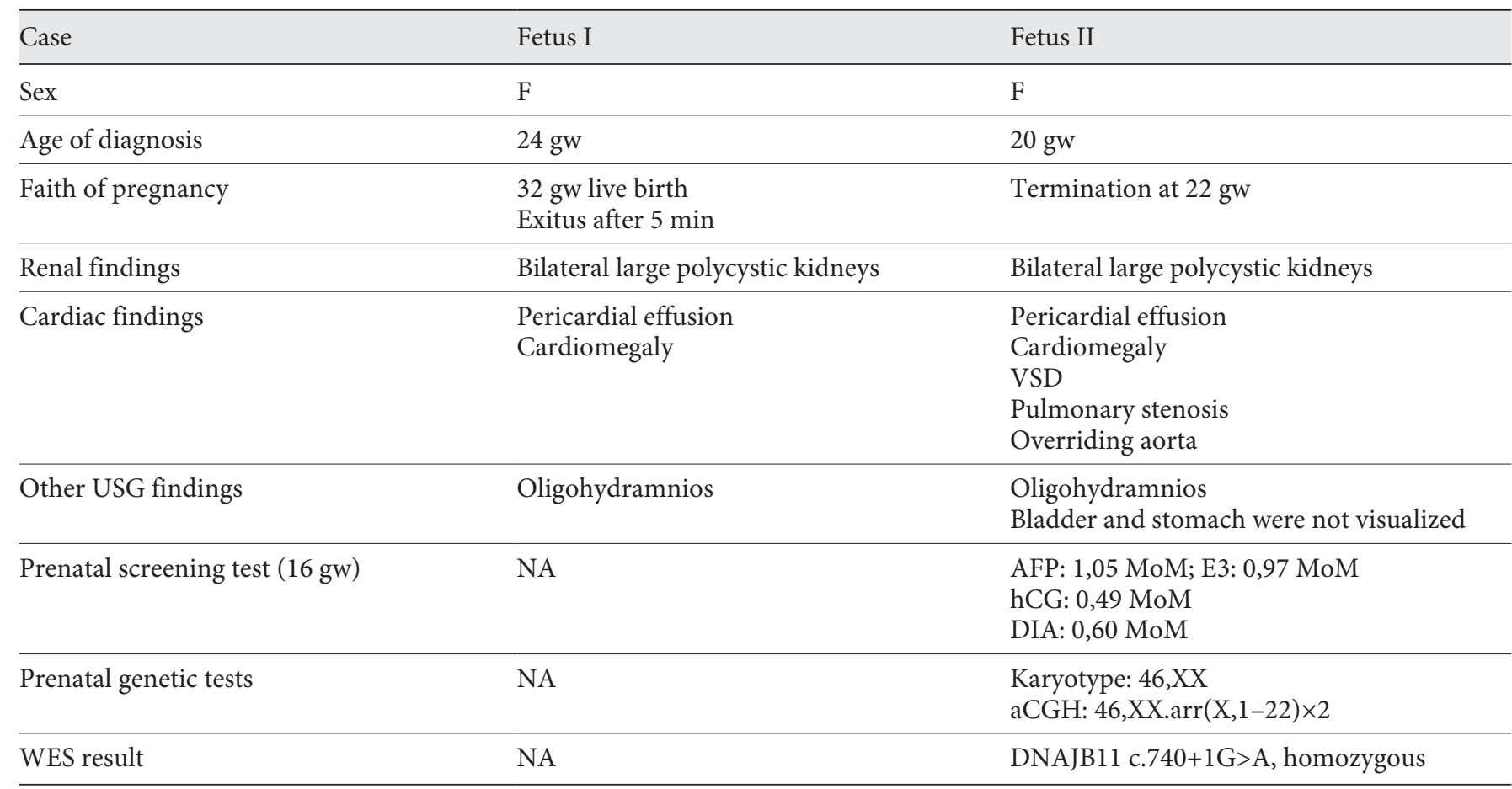

NA, not available; gw, gestational weeks; VSD, ventricular septal defect.

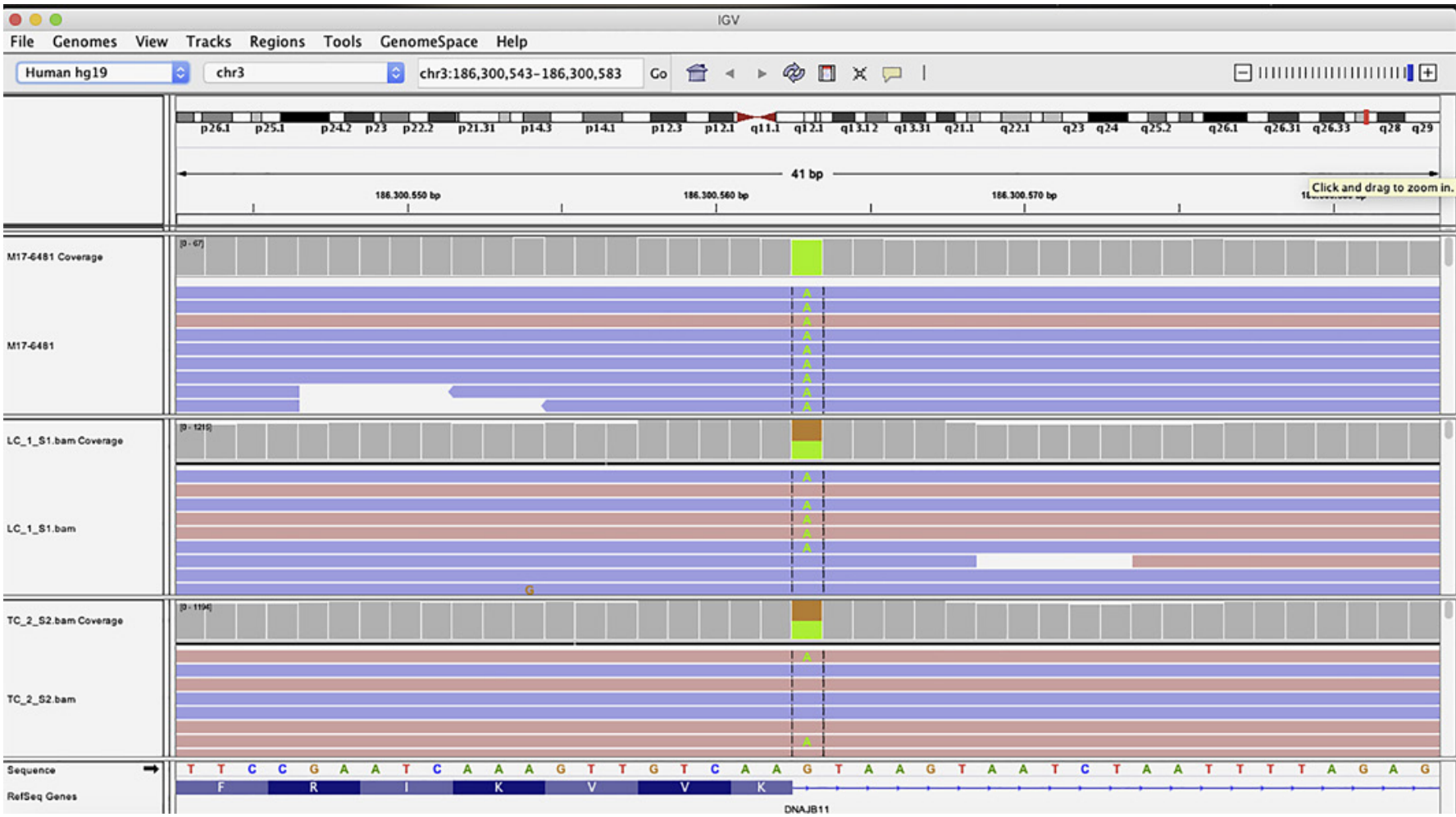

Fig. 3. DNAJB11 (NM_016306) gene exon 7 - intron boundary. A novel homozygous c.740+1G>A variant in the fetus and heterozygous c.740+1G>A variant in the parents are shown. 
Table 2. Clinical findings of parents and comparision of DNAJB11-related ADPKD cases

\begin{tabular}{|c|c|c|c|}
\hline & Mother & Father & Reported cases $(n / 23)$ \\
\hline Age & 25 ys & 32 ys & Ranges between 19 and 80 ys \\
\hline Nephrolithiasis & + & + & $2 / 23$ \\
\hline Hearing loss & - & Bilateral mild sensorineural hearing loss & $1 / 23$ \\
\hline Renal function & $\begin{array}{l}\text { Normal } \\
\text { Urine erithroctyte }(+)\end{array}$ & Normal & $\begin{array}{l}\text { Renal insufficiency in their } \\
6 \text { th-7th decade }(7 / 20)\end{array}$ \\
\hline Renal imaging & Multiple bilateral cysts (largest $18 \mathrm{~mm}$ ) & Normal & $\begin{array}{l}\text { 20/23 multiple renal cysts } \\
1 / 20 \text { no cysts }(19 \text { ys }) \\
2 / 20 \text { NA }\end{array}$ \\
\hline Hepatic imaging & 2 cysts ( 8 and $12 \mathrm{~mm})$ & $\begin{array}{l}\text { Hepatomegaly } \\
\text { Fatty liver }\end{array}$ & $\begin{array}{l}9 / 23 \text { multiple cysts } \\
11 / 23 \text { no cysts } \\
3 / 23 \text { NA }\end{array}$ \\
\hline Cranial imaging & $\begin{array}{l}\text { Bilateral increased Virchow-Robin } \\
\text { spaces (sublentiform area) }\end{array}$ & $\begin{array}{l}\text { Bilateral increased Virchow-Robin } \\
\text { spaces (sublentiform area) }\end{array}$ & NA \\
\hline Other findings & Unilateral breast cyst ( $5 \mathrm{~mm})$ & - & $\begin{array}{l}\text { Breast cancer } \\
\text { Colorectal cancer } \\
\text { Metanephric adenoma } \\
\text { Glioblastoma } \\
\text { Parathyroid adenoma } \\
\text { Hypertrophic cardiomyopathy } \\
\text { Epilepsy }(2 / 23) \\
\text { Parkinson disease } \\
\text { Gout }(3 / 23) \\
\text { Peptic ulcer }\end{array}$ \\
\hline
\end{tabular}

NA, not available; ys, years; +, present; -, absent.

(Fig. 3). This variant was not reported previously in HGMD or ClinVar databases and was not present in population studies. The variant was predicted as disease causing via MutationTaster and alterating donor site and most probably affecting splicing via HSF [Desmet et al., 2009]. Segregation analysis revealed that parents carried the mutation in heterozygous state (Fig. 3). Parents were evaluated for ADPKD. Both had no complaints but nephrolithiasis. Imaging studies revealed multiple bilateral renal cysts, hepatic cysts, and bilateral breast cysts in the mother. In the father, only fatty liver, hepatomegaly, and bilateral sensorineural hearing loss was detected. Cranial imaging showed bilateral increased Virchow-Robin spaces in both. Clinical findings of the parents are summarized in Table 2.

\section{Discussion}

The DNAJB11 gene is located in chromosome $3 \mathrm{q} 27.3$ and spans 11 exons. It encodes a soluble glycoprotein that functions as a co-chaperone of $\mathrm{BiP}$. This protein is a
HSP70 molecular chaperon located in ER and has an important role in folding of many proteins. Protein folding errors cause many human diseases due to resulting in nonfunctional misfolded proteins and ER stress. DNAJB11 was associated with loss of maturation and appropriate localization of $P K D 1$, which regulates multiple signaling pathways to maintain normal renal tubular structure and function with PKD2 [Cornec-Le Gall et al., 2013; Song et al., 2009].

Cornec-Le Gall et al. [2018] previously reported DNAJB11 monoallelic mutations in a form of late-onset PKD (OMIM \#618061). The study suggested that DNAJB11 mutations result in impaired maturation of $P K D 1$ causing cystogenesis. DNJAB11-related ADPKD patients have distinct features compared to the other ADPKD forms. Liver cysts were present in approximately half of the patients. All patients in the study had small renal cysts which did not lead to enlarged kidneys and were not detected in younger individuals. End-stage renal disease and renal failure was an important cause of morbidity in the patients in their 6th and 7th decade [Cornec-Le Gall 
et al., 2018]. In our study, the parents carry the mutation in a heterozygous state; the 25-year-old mother had multiple cysts in kidneys and liver. The father's renal and hepatic MRI were normal, at the age of 32. Renal functions also were normal. The parents' findings were compatible with the cases carrying DNAJB11 mutations reported previously, as stated in Table 2 [Cornec-Le Gall et al., 2018]. Interestingly, cranial MRI revealed bilateral increased Virchow-Robin spaces, which indicate small vessel disease in both parents as a novel finding. In a recent study, cerebral microbleeds were reported in $27.3 \% \mathrm{PKD}$ patients without enlarged perivascular space [Tsai et al., 2020]. In another study, aberrant regulation of Notch3 pathway was reported which may be a cause of enlarged perivascular space and was correlated with rapidly growing cysts in PKD mouse models and patients [Idowu et al., 2018]. In the study of Hunyh et al. [2020], aneurysms in thoracic aorta and intracranial vessels in 4 different families with DNAJB11 heterozygous mutation are reported. We can conclude that MRI findings should be considered in ADPKD patients, especially having DNAJB11 mutations.

ARPKD is a more severe, early-onset PKD form. Usually the patients are diagnosed perinatally or at childhood. PKHD1 and DZIP1L mutations are the common causes of ARPKD. Also many more genes are related to PKD causing ciliopathies [Bergmann et al., 2018]. Widespread usage of next-generation sequencing strategies allowing analyses of multiple genes at once provide more information about PKD. It is reported that biallelic mutations in PKD1, a well-known ADPKD gene, result in more severe and early-onset PKD [Vujic et al., 2010; Al-Hamed et al., 2019]. In the present study, 2 fetuses in the same family were diagnosed as PKD at antenatal period, and we were able to perform WES in one of them. The DNAJB11 gene c. $740+1 \mathrm{G}>\mathrm{A}$ variant, which was the only variant related to $\mathrm{PKD}$ previously, was detected in a homozygous state, and the parents are heterozygous carriers of the mutation. This variation is predicted to disrupt the donor splice site in 7 th exon-intron boundary. It is predicted to be a deleterious mutation according to clinical compliance, segregation and in silico analyses. The mutation also is not present in any population studies and in our in-house database. Functional splicing analysis could not be performed due to the absence of fresh tissue of the fetus.

While preparing this paper, Jordan et al. [2021] reported a fetus diagnosed with renal-hepatic-pancreatic dysplasia with a homozygous c.600-2A $>C$ splice site mutation in DNAJB11. The mutation was shown to cause tortuous primary cilia in renal tubules in the fetus. Renal and cardiac features of the fetus were smiliar to our cases; however, pancreatic and hepatic findings were not mentioned in the autopsy report [Jordan et al., 2021].

In conclusion, this study reveals that DNAJB11 biallelic mutations may cause an antenatal severe form of ARPKD and contributes to understanding DNAJB11-related ADPKD phenotytpes. The possibility of ARPKD due to biallelic mutations in ADPKD genes should be considered in genetic counseling.

\section{Statement of Ethics}

The study was approved by the Local Ethical Committee, conducted in adherence with the Declaration of Helsinki and informed written consent was obtained from the parents.

\section{Conflict of Interest Statement}

The authors have no conflicts of interest to declare.

\section{Funding Sources}

No specific funding was received for this study.

\section{Author Contributions}

Esra Arslan Ateş and Ayberk Türkyılmaz performed the research. Esra Arslan Ateş and Ceren Alavanda designed the research study. Esra Arslan Ateş, Ayberk Türkyılmaz, Kenan Delil, Mehmet Ali Söylemez, Bilgen Bilge Geçkinli, Pınar Ata, and Ahmet Arman analyzed the clinical and genetic data. Esra Arslan Ateş wrote the paper.

\section{References}

Al-Hamed MH, Alsahan N, Rice SJ, Edwards N, Nooreddeen E, Alotaibi M, et al. Bialleleic PKD1 mutations underlie early-onset autosomal dominant polycystic kidney disease in Saudi Arabian families. Pediatr Nephrol. 2019;34(9):1615-23.
Alzarka B, Morizono H, Bollman JW, Kim D, Guay-Woodford LM. Design and Implementation of the Hepatorenal Fibrocystic Disease Core Center Clinical Database: A Centralized Resource for Characterizing Autosomal Recessive Polycystic Kidney Disease and Other
Hepatorenal Fibrocystic Diseases. Front Pediatr. 2017;5:80.

Bergmann C, Guay-Woodford LM, Harris PC, Horie S, Peters DJM, Torres VE. Polycystic kidney disease. Nat Rev Dis Primers. 2018; 4(1):50. 
Cornec-Le Gall E, Audrézet MP, Chen JM, Hourmant M, Morin MP, Perrichot R, et al. Type of PKD1 mutation influences renal outcome in ADPKD. J Am Soc Nephrol. 2013;24(6): 1006-13.

Cornec-Le Gall E, Olson RJ, Besse W, Heyer CM, Gainullin VG, Smith JM, et al. Monoallelic Mutations to DNAJB11 Cause Atypical Autosomal-Dominant Polycystic Kidney Disease. Am J Hum Genet. 2018;102(5):832-44.

Desmet FO, Hamroun D, Lalande M, CollodBéroud G, Claustres M, Béroud C. Human Splicing Finder: an online bioinformatics tool to predict splicing signals. Nucleic Acids Res. 2009;37(9):e67.

Huynh VT, Audrézet M-P, Sayer JA, Ong AC, Lefevre S, Le Brun V, et al. Clinical spectrum, prognosis and estimated prevalence of DNAJB11-kidney disease. Kidney International. 2020;98(2):476-87.
Idowu J, Home T, Patel N, Magenheimer B, Tran PV, Maser RL, et al. Aberrant Regulation of Notch3 Signaling Pathway in Polycystic Kidney Disease. Sci Rep. 2018;8(1):3340.

Jordan P, Arrondel C, Bessières B, Tessier A, Attié-Bitach T, Guterman S, et al. Bi-allelic pathogenic variations in DNAJB11 cause Ivemark II syndrome, a renalhepatic-pancreatic dysplasia. Kidney Int. 2021;99(2):405-9.

Mochizuki T, Wu G, Hayashi T, Xenophontos SL, Veldhuisen B, Saris JJ, et al. PKD2, a gene for polycystic kidney disease that encodes an integral membrane protein. Science. 1996; 272(5266):1339-42.

Porath B, Gainullin VG, Cornec-Le Gall E, Dillinger EK, Heyer CM, Hopp K, et al. Mutations in GANAB, Encoding the Glucosidase IIa Subunit, Cause Autosomal-Dominant Polycystic Kidney and Liver Disease. Am J Hum Genet. 2016;98(6):1193-207.
Song X, Di Giovanni V, He N, Wang K, Ingram A, Rosenblum ND, et al. Systems biology of autosomal dominant polycystic kidney disease (ADPKD): computational identification of gene expression pathways and integrated regulatory networks. Hum Mol Genet. 2009; 18(13):2328-43.

Suwabe T, Shukoor S, Chamberlain AM, Killian JM, King BF, Edwards M, et al. Epidemiology of Autosomal Dominant Polycystic Kidney Disease in Olmsted County. Clin J Am Soc Nephrol. 2020;15(1):69-79.

Tsai LK, Chen PL, Tsai HH, Chen YF, Wu PC, Jeng JS, et al. Cerebral Microbleeds in Autosomal Dominant Polycystic Kidney Disease. J Stroke. 2020;22(1):153-6.

Vujic M, Heyer CM, Ars E, Hopp K, Markoff A, Orndal C, et al. Incompletely penetrant PKD1 alleles mimic the renal manifestations of ARPKD. J Am Soc Nephrol. 2010;21(7):1097102 